\title{
An Enhanced Power Save Mode for IEEE 802.11 Station in Ad Hoc Networks*
}

\author{
Yeonkwon Jeong ${ }^{1}$, Jongchul Park ${ }^{1}$, Joongsoo Ma ${ }^{1}$, and Daeyoung Kim² \\ ${ }^{1}$ School of Engineering, Information and Communications University, \\ Daejeon, 305-714, KOREA \\ \{ykwjeong, jcpark, jsma\}@icu.ac.kr \\ ${ }^{2}$ Dept. of InfoCom Engineering, Chungnam National University, \\ Daejeon, 305-764, KOREA \\ dykim@enu.ac.kr
}

\begin{abstract}
The IEEE 802.11 standard requires that a beacon station has to stay in awake after generating a beacon frame to serve probe request. This requirement make a reason to consume valuable power of beacon station even though there are no frame exchanges between stations or there exists only one station in the ad hoc network. This paper proposes an ePSM(Enhanced Power Save Mode) to reduce effectively the power consumption of beacon station in stand-by state. We also develop a power consumption model and show that the ePSM can save $73 \%$ energy at beacon station per a BI(Beacon Interval) with measurement results.
\end{abstract}

\section{Introduction}

Today wireless LANs based on the IEEE 802.11 standard are widely used at offices and homes. Stations can form a network using one of the two modes of operation specified in the standard: the infrastructure mode and the ad hoc mode.

The infrastructure mode is suitable for stations to connect to the enterprise network and the Internet. In this mode, one station must be designated as an AP(Access Point). The AP is the smart master and other stations follow the control of the AP. Stations must communicate via the AP. Wireless LANs(Local Area Network) use the infrastructure mode.

On the other hand, the ad hoc mode allows stations to form an ad hoc network in a peer-to-peer manner. Stations communicate to each other directly without requiring any central controller such as an AP. Therefore networking can take place anywhere the stations meet. The ad hoc mode is useful in connecting computers, peripheral devices and multimedia appliances at home or offices. It is particularly useful when notebook computers or PDAs are joined together to play network games. Since there is no AP in the middle to route packets between stations, the throughput can be much higher with a less delay. However these stations are often portable and rely on their battery-supplied power. Therefore they must save power, particularly during the stand-by periods in which they are not sending or receiving any packet.

\footnotetext{
* This research was supported by University IT Research Center Project.
} 
So far the majority of the IEEE 802.11 market has been in the infrastructure mode, and it is not well known how effective the standard method of saving power in the ad hoc mode is. There are a few research activities to conserve power consumption by considering characteristics from MAC(Medium Access Control) to application layer. Their researches focus on measurement by experiments, and analyze those results with existing WaveLAN at infrastructure mode[2], or Lucent IEEE802.11 at ad hoc mode[3]. Other researches measure power consumption at application oriented view such as email[4] and web[5]. The last approaches use a coordinator which stay in awake continuously[6], or a soft-state timer for transition between active and sleep mode[7].

In this paper, we propose an ePSM(Enhanced Power Save Mode) to reduce effectively the power consumption of beacon station in stand-by state. We also develop a power consumption model of the IEEE 802.11 ad hoc mode operation. Our model is based on physical measurements. We place our emphasis in investigating the effectiveness of ad hoc mode power saving features during the stand-by periods. This environment is important because we expect that the IEEE802.11 ad hoc mode will be most popular in offices and homes, where a few stations are connected and left connected for a whole day, waiting for occasional communication works to occur.

The remainder of this paper is organized as follows. Section II reviews the IEEE 802.11 standard in view of PSM and ad hoc mode. Section III shows the observed problem of current standard and proposes new ePSM to resolve this issue. Section IV presents numerical and experimental results. Finally Section V concludes this paper.

\section{Overview of the IEEE 802.11}

We will review ad hoc network setup and maintenance procedures in the aspect of the beacon frame, scanning procedures, and PSM of IEEE 802.11.

\subsection{Beacon Frame}

In an ad hoc network, the generation of beacon frame is completely distributed among the mobile stations of the network. Each station will attempt a back-off for a random time to send a beacon frame every BI(Beacon Interval). In the random time, if a station hears a beacon it cancels its transmission. A beacon frame contains ad hoc network configuration parameters. Those are not only timing information for synchronization, but also network identifier, a BI, ATIM(Announce Traffic Indication Message) window size, and the data rates that can be supported.

\subsection{Scanning Procedures}

Scanning procedures are necessary when station want to join an already existing an ad hoc network. If the scanning procedure does not find any network, the station may start with the creation of a new network. The scanning procedures can be either passive or active. In a passive scanning the station only listens to the channel for 
hearing a beacon frame. However, the station of active scanning generates probe request frames, and processes probe response frames received. Only the station that generated the last beacon frame will respond to a probe request, in order to avoid the waste of bandwidth with repetitive control frames.

\subsection{Power Management}

A station may be in one of two different power states: awake which is fully powered or doze which is not able to transmit or receive frames because of low power. Stations having frames destined to a power-conserving station are first announced ATIM frame to wake up one or more doze stations during ATIM window. A station operating in the PSM listens to these announcements and, based on them, decides whether it has to remain awake or not.

\section{Observed Problem and Solution}

\subsection{Observed Problem}

The IEEE 802.11 standard requires that the station which send a beacon frame shall remain in the awake state until the end of the next ATIM[1]. Basically, the reason of this restriction comes from an intention to use active scanning as a default mode. Only the station that generated the last beacon frame will respond to a probe request, in order to avoid the waste of bandwidth with repetitive control frames.

However, the standard prevent from conserving the power consumption of beacon station even though it is possible. The standard is missing the following cases when the network is ad hoc. One of possible examples is when a beacon station is being stand-by. Stand-by means that a station does not have any user traffics to transmit or receive frames. The other is that as a subset of previous case, an ad hoc network has only one member being stand-by. In both cases the station should always stay in awake to meet the IEEE 802.11 requirements without doing anything.

If the standard permits beacon station being stand-by to go doze state, the power consumption of beacon station can be saved as much as $\Delta(m W)$. The power gains is $\Delta(m W)=\int_{T_{\text {doze }}(t)}\left(p_{\text {awake }}-p_{\text {doze }}\right) d t$. Where, $T_{\text {doze }}(t)$ is a time period over BIATIM window. $p_{\text {awake }}$ and $p_{\text {doze }}$ are the power required for station to be in awake and doze state.

\subsection{Enhanced Power Save Mode}

In this paper we propose new ePSM mechanism to improve power consumption of beacon station in stand-by state. ePSM is that

- Ad hoc network use the passive scanning instead of the active scanning of IEEE 802.11 standard. 
- A beacon station may go to doze state if the station does not have transmit or receive frames. Like a Fig. 1, the beacon node of IEEE 802.11 shall remain awake during one BI irrespective of having transmitting or receiving frames. But we permit this node to go doze in that case.

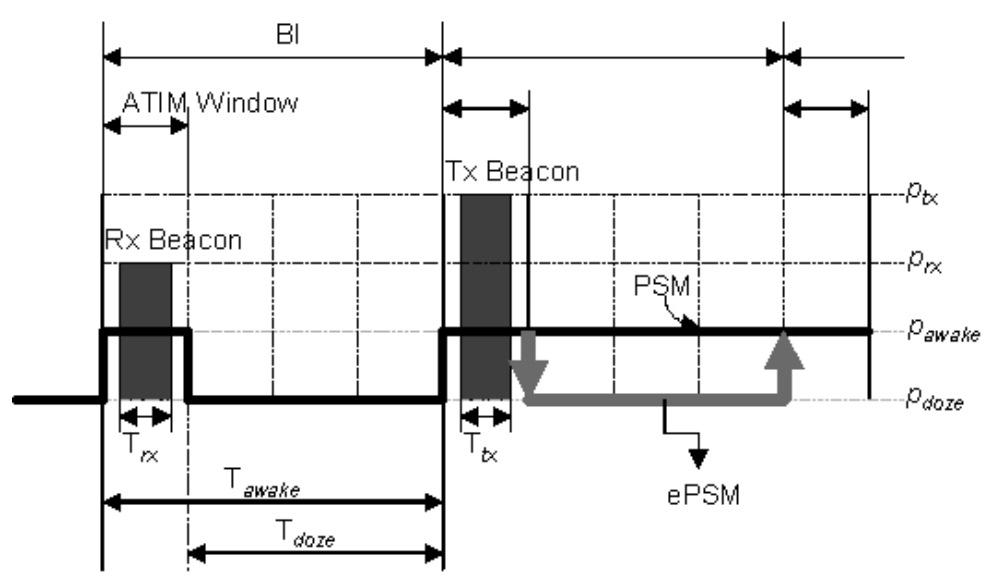

Fig. 1. The operation of PSM and ePSM

\section{Numerical and Experimental Results}

\subsection{Numerical Results}

We will use APC(Average Power Consumption) and APG(Average Power Gains) as the comparison parameters of different PSMs. APC is the averaged amount of power required to operate during one $\mathrm{BI}$ in an ad hoc network having $N$ stand-by stations. The power consumption of any station is expressed by the sum of these values dependent on the state of the station:

$$
\begin{gathered}
\text { transmit: } \int_{T_{t x}(t)} p_{t x} d t \text {, receive: } \int_{T_{r x}(t)} p_{r x} d t, \\
\text { awake: } \int_{T_{\text {awake }}(t)} p_{\text {awake }} d t \text {, doze: } \int_{T_{\text {doze }}(t)} p_{\text {doze }} d t
\end{gathered}
$$

In the non-PSM, an ad hoc network consists of a beacon transmit station and the beacon receive stations of $N-1$. All station of non-PSM remains always awake state and the APC of non-PSM is sum of transmit, receive and awake state power. In the PSM, the beacon station is awake and the other stations of ad hoc network are doze. The APC of PSM is calculated by reduced value from non-PSM as much as the number of doze state stations. In the ePSM, all station is doze state irrespective of 
beacon generation. So we can minimize the energy consumption among these three types.

APG is a relative value which is reducible amount from the power consumption of non-PSM. Hence the APG is zero for non-PSM. However APG for PSM is $\left(\frac{N-1}{N}\right) \Delta$, and ePSM is $\Delta$. If the number of node $N$ is increasing infinitely, the APG of PSM will converge on that of ePSM such as $\lim _{N \rightarrow \infty}\left(\frac{N-1}{N}\right) \Delta \simeq \Delta$.

Table 1 shows the APC and APG of an ad hoc network having $N$ stand-by stations for 3 type mechanisms: non-PSM, PSM, and ePSM.

Table 1. The APC and APG for non-PSM, PSM, and ePSM

\begin{tabular}{|c|c|c|}
\hline Types & APC & APG \\
\hline \multirow[t]{2}{*}{$\begin{array}{l}\text { non- } \\
\text { PSM }\end{array}$} & $\left\{\begin{array}{l}\int_{T_{t x}(t)} p_{t x} d t+(N-1) * \int_{T_{r x}(t)} p_{r x} d t \\
+N * \int_{T_{\text {awake }}(t)} p_{\text {awake }} d t\end{array}\right.$ & 0 \\
\hline & $N$ & \\
\hline PSM & $n o n_{-} P S M-\left(\frac{N-1}{N}\right) \Delta$ & $\left(\frac{N-1}{N}\right) \Delta$ \\
\hline ePSM & $n o n_{-} P S M-\Delta$ & $\Delta$ \\
\hline
\end{tabular}

Fig. 2 shows the transition of APG when $\Delta$ is normalized to 1 and the number of stations participated in ad hoc network are increasing. As you can see the results, ePSM is effective when the number of stations is small than any other mechanisms. This is a significant gain because there would be only one station when the ad hoc network is started, and there would be many applications where two or three stations are connected.

\subsection{Experimental Results}

We conduct measurement to get the practical power values of Table 1 such as $p_{t x}, p_{r x}$, $p_{\text {avake, }}$ and $p_{\text {doze. }}$ The circuit and methodology are closely based on the experiments reported in [3]. We are using SAMSUNG SENSE T10 laptop computers running Linux RedHat8.0 with Cisco Aironet 350 series client adapter and PCCextend 140A 


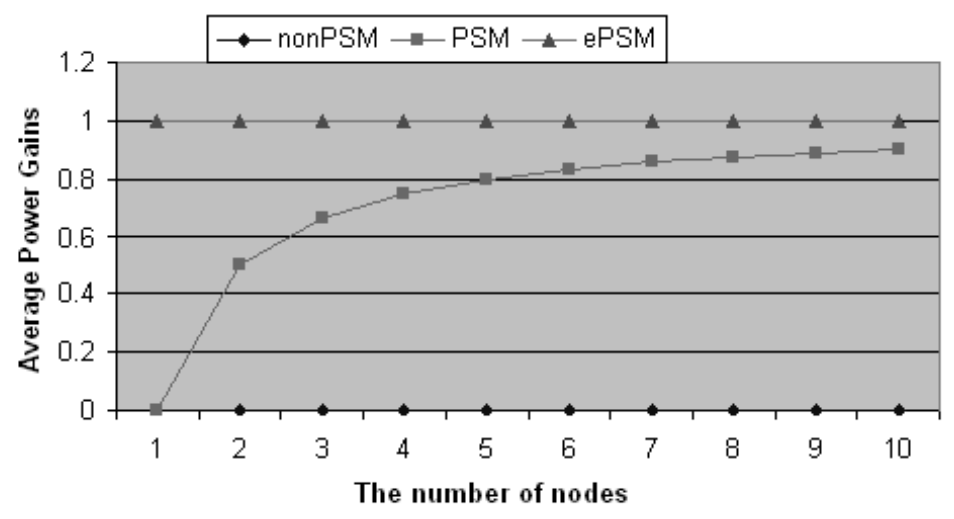

Fig. 2. The APG of non-PSM, PSM, and ePSM in an ad hoc network

card bus extender. The BI and ATIM window values applied to this measurement are $100 \mathrm{msec}$ and $5 \mathrm{msec}$ respectively. Transmit power is $100 \mathrm{~mW}$ and input voltage is 4.8165 .

Measurement results show that awake state station keeps going on idle phase for a BI continuously. There is also another interesting point on the doze state station. After ATIM window, dose state station goes through sleep phase, and reaches warm-up phase to prepare a transition from dose to awake state. Warm-up takes for $13.14 \mathrm{msec}$. Table 2 is the summary of measured average currents and power consumptions in each phase.

Table 2. Aironet 350 NIC characteristics

\begin{tabular}{|c|c|c|}
\hline Phase & Current $(\mathrm{mA})$ & Power(mW) \\
\hline Transmit & 270.83 & $1,304.476$ \\
\hline Receive & 198.17 & 954.507 \\
\hline Idle & 164.12 & 790.47 \\
\hline Sleep & 35.07 & 168.91 \\
\hline Warm-up & 105.75 & 509.35 \\
\hline
\end{tabular}

Let's calculate the absolute power gains $\Delta(\mathrm{mW})$ using the measurement results. Our interest is the difference of power consumption between awake and doze state station during the interval of BI-ATIM window.

$$
\begin{gathered}
P_{\text {awake }}(m W)=790.47 *(B I-\text { ATIM Window }) \\
P_{\text {dose }}(m W)=168.91 * T_{\text {Sleep }}+509.35 * T_{\text {Warmup }}
\end{gathered}
$$


Therefore, the $\Delta(\mathrm{mW})$ is 54577.1186 . A doze state station uses the small portion $27 \%$ of awake state station power. If we permit beacon station being stand-by to go doze state, ePSM can achieve the energy saving effect of $73 \%$ at that beacon station per one BI.

\section{Conclusion}

In this paper, we point out the weakness of IEEE 802.11 PSM when the PSM is applied to ad hoc network environment. And this paper proposes new ePSM to enhance the power consumption of beacon station in stand-by state. We show that the ePSM can save the $73 \%$ of energy at beacon generation station per a BI using measurement results. Our proposed method is effective when the number of stations is small. This is a significant gain because there would be only one station when the ad hoc network is started, and there would be many applications where two or three stations are connected in home or office environments.

\section{References}

1. IEEE Standard 802.11b, Part 11: Wireless LAN Medium Access Control(MAC) and Physical Layer(PHY) specifications : High Speed Physical Layer Extension in the $2.4 \mathrm{GHz}$ Band, 1999.

2. R. Kravets, and P. Krishnan, "Power Management Techniques for Mobile Communication", Proc. of MOBICOM 1998, Oct. 1998.

3. L. M. Feeney, and M. Nilsson, "Investigating the Energy Consumption of a Wireless Network Interface in an Ad Hoc Networking Environment", Proc. of INFOCOM 20001, Apr. 2001.

4. M. Stemm, and R. H. Katz, "Measuring and Reducing Energy Consumption of Network Interfaces in Handheld Devices", IEICE Trans. on Fundamentals of Elec., Comm., and Computer Science, Aug. 1997

5. P. Gauthier, D. Harada, and M. Stemm, "Reducing Power Consumption for the Next Generation of PDAs: It's in the Network Interface!”, Proc. of MoMuc 1996, Sep. 1996

6. B. Chen, K. Jamieson, H.Balakrishnan, and R. Morris, "Span : An energy-efficient coordination algorithm for topology maintenance in ad hoc wireless networks", Proc. of MOBICOM 2001, Jul. 2001

7. R. Zheng, R. Kravets, "On-demand Power Management for Ad Hoc Networks", Proc. of INFOCOM, 2003 